\title{
ON THE REPRESENTATION OF CARDINAL ALGEBRAS BY DIRECTED SUMS
}

\author{
BY \\ A. BRUCE CLARKE
}

1. Introduction. The term cardinal algebra was introduced by A. Tarski in his treatise [4] to signify an algebraic system closed under an operation of countable addition satisfying certain axioms abstracted from the common properties of such diverse systems as the algebra of cardinal numbers, the algebra of sets, relation algebras, etc. In [4] Tarski studied the arithmetical properties of such systems, methods of their construction, and their connection with other algebraic systems. Some of these results are summarized in $\$ 2$.

It is the purpose of this paper to develop a representation theory for such algebras in some sense analogous to the classical semisimple ring theory. It is desired to show how general cardinal algebras can be built up in a unique fashion from special and more elementary types of algebras. Then by adding some fairly mild restrictions these special algebras can in turn be described more or less completely. In doing this it will be necessary to introduce some new methods of algebraic construction. The methods used appear to be powerful enough to deserve further investigation, and the present discussion suggests several problems which still remain to be settled.

The classical method of Birkhoff on representation of algebras as direct products does not appear to be particularly fruitful here.

Since a cardinal algebra is, in particular, a partially ordered set, it seems natural to attempt to build representations in terms of the order structure, as well as along more classical lines. Consequently in $\$ 3$ an operation of ordered or directed sums of cardinal algebras is defined (3.3) and necessary and sufficient conditions (3.5) are given for this operation to yield a cardinal algebra. The main theorem of this section (3.14) states that every cardinal algebra has a unique representation as a linear directed sum of algebras not further decomposable in this fashion. In $\$ 4$ it is shown that, under one fairly natural restriction, these indecomposable "summands" must be either idemmultiple algebras or simple algebras (see $\$ 2$ for definitions).

The structure of idemmultiple cardinal algebras is fairly well knownalthough not as well as one would like. Every such algebra is isomorphic to an algebra of sets under the operation of set-theoretical union. The isomorphism is given by

$$
a \rightarrow\{x \mid x \geq a\} .
$$

The situation with regard to simple cardinal algebras is not so satisfactory.

Received by the editors June 17, 1957. 
However theorem (4.7) gives a fairly weak condition under which a simple algebra is a subalgebra of the nonnegative reals.

$\S 5$ is devoted to the definition and discussion of an operation on cardinal algebras which is, in a certain sense, a generalization of both a directed sum and a direct product. This operation is used in $\$ 6$ to obtain a representation theory for a still larger class of cardinal algebras.

2. Notations and known results. Although some acquaintance by the reader with the treatise of Tarski [4] on cardinal algebras must be assumed, this opportunity will be taken to summarize briefly those definitions and basic results from that work to which specific reference will be made later. The numbering on the right-hand side of the page refers to the numbering system in [4].

(2.1) By a CARDINAL ALGEBRA is meant an algebraic system

$$
\mathfrak{A}=\left\langle A,+, \sum\right\rangle
$$

satisfying the following postulates.

II $\quad A$ is closed under countably infinite addition $\sum$;

III $\quad \sum_{i<\infty} a_{i}=a_{0}+\sum_{i<\infty} a_{i+1}$;

IV

$$
\sum_{i<\infty}\left(a_{i}+b_{i}\right)=\sum_{i<\infty} a_{i}+\sum_{i<\infty} b_{i}
$$

$\mathrm{V}$

there exists a zero element "0" such that

$a+0=a=0+a$ for each $a \in A$;

VI

$$
a+b=\sum_{i<\infty} c_{i}
$$

implies the existence of elements $a_{i}, b_{i} \in A$ for $i<\infty$, such that

$$
a=\sum_{i<\infty} a_{i}, \quad b=\sum_{i<\infty} b_{i}, \quad \text { and } c_{i}=a_{i}+b_{i} \text { for } i<\infty ;
$$

VII $a_{i}=b_{i}+a_{i+1}$ for each $i<\infty$

implies the existence of an element $c \in A$ such that

$$
a_{n}=c+\sum_{i<\infty} b_{n+i} \text { for each } n<\infty .
$$

These postulates imply that $\sum$ is a generalization of + , and that the algebra is unrestrictedly commutative and associative.

From now on the term cardinal algebra will be abbreviated by C.A.

In any C.A. a partial ordering $\leqq$ is introduced by

$$
a \leqq b \text { if and only if } b=a+c \text { for some } c \in A \text {. }
$$

One has the following arithmetical theorems: 
(2.3) If $a_{1}+a_{2}=b_{1}+b_{2}$, then there exist elements $c_{1}, c_{2}, c_{3}, c_{4} \in A$ such that $a_{1}=c_{1}+c_{2}, a_{2}=c_{3}+c_{4}, b_{1}=c_{1}+c_{3}, b_{2}=c_{2}+c_{4}$.

(2.4) If $a+c=b+c$, then there exist elements $a^{\prime}, b^{\prime}, d \in A$ such that $a=a^{\prime}+d$, $b=b^{\prime}+d, c=a^{\prime}+c=b^{\prime}+c$. (This is a modified cancellation law.)

$$
\text { If } \sum_{i<n} a_{i} \leqq b \text { for every } n<\infty \text {, then } \sum_{i<\infty} a_{i} \leqq b .
$$

$$
\text { If } n \text { is an integer, } 0<n<\infty \text {, and } n a \leqq n b \text {, then } a \leqq b .
$$

The concept of an idemmultiple element or C.A. plays an important role in the sequel.

An element $a$ is said to be IDEmmultiple if $a+a=a . A$ C.A. is said to be IDEMMULTIPLE if each of its elements is idemmultiple.

One has the theorem

(2.7) An element $a$ is idemmultiple if and only if $p a=q a$ for some distinct nonnegative integers $p$ and $q$.

A subset $B \subset A$ is said to be an ideal in $\mathfrak{A}$ if it is closed under + and $\sum$ and if $a \leqq b \in B$ implies that $a \in B$. If $X \subset A$, the closure $I(X)$ represents the smallest ideal containing $X$. For single element sets $I(a)$ will be used in place of $I(\{a\})$. If $B$ is an ideal the relation $\equiv_{B}$ defined by: $a \equiv_{B} b$ if $a+c=b+c^{\prime}$ for some elements $c, c^{\prime} \in B$, is an infinitely additive equivalence relation. The algebra of equivalence classes $\mathfrak{A} / B$ is also a C.A. The class of all ideals in a C.A. is itself an idemmultiple C.A. under the operation $\sum_{i<\infty} B_{i}=I\left(\cup_{i<\infty} B_{i}\right)$. This algebra is denoted by $\Im(\mathfrak{A})$. A simple C.A. $\mathfrak{A}$ is a C.A. having no ideals other than $\{0\}$ and $A$ itself.

In the following the usual set-theoretic notations will be used, the empty set being denoted by $\varnothing$.

3. Directed sums of cardinal algebras. The notion of a directed sum of partially ordered sets over a partially ordered set is known from the literature $[1 ; 3]$. Roughly speaking, one replaces each member $a$ of a partially ordered set $A$ by a partially ordered set $B_{a}$ and obtains in this manner a new partially ordered set. More precisely, suppose $A$ is partially ordered by the relation $\leqq$, and for each $a \in A$ suppose $B_{a}$ is partially ordered by a relation $\leqq_{a}$. Let $C$ be the set of all ordered pairs $\langle a, b\rangle$ with $a \in A$ and $b \in B_{a}$. For $\langle a, b\rangle,\left\langle a^{\prime}, b^{\prime}\right\rangle$ $\in C$ define $\left.\langle a, b\rangle \leqq a^{\prime}, b^{\prime}\right\rangle$ in case either $a \leqq a^{\prime}, a \neq a^{\prime}$ or else $a=a^{\prime}, b \leqq{ }_{a} b^{\prime}$. The relation $\leqq$ partially orders the set $C$, and the system $\left\langle C, \leqq^{\prime}\right\rangle$ is called the directed sum of the systems $\left\langle B_{a}, \leqq a\right\rangle$ over the system $\langle A, \leqq\rangle$; in symbols

$$
\left\langle C, \leqq^{\prime}\right\rangle=\sum_{(a, A, \leqq)}\left\langle B_{a}, \leqq_{a}\right\rangle .
$$

Since a C.A. is in particular a partially ordered set, it seems natural to attempt to define an operation on C.A.'s analogous to this operation of directed addition of partially ordered sets.

Consider C.A.'s $\mathfrak{A}=\left\langle A,+, \sum\right\rangle$ and $\mathfrak{B}_{a}=\left\langle B_{a},+_{a}, \sum_{a}\right\rangle$ for each $a \in A$. The operation + induces a partial ordering $\leqq$ of the set $A$ while, for each $a \in A$, 
the operation $+_{a}$ induces a partial ordering $\leqq_{a}$ of the set $B_{a}$. Let $\left\langle C, \leqq^{\prime}\right\rangle$ be the directed sum of the systems $\left\langle B_{a}, \leqq_{a}\right\rangle$ over the system $\langle A, \leqq\rangle$. It is desired to define operations

$$
\mp \text { and } \bar{\sum}
$$

over the set $C$ such that the algebra

$$
\mathfrak{c}=\langle C, \mp, \bar{\Sigma}\rangle
$$

is a C.A. and $\leqq^{\prime}$ is the partial ordering of $C$ induced by the operation $\mp$. This condition alone would not determine the operations

$$
\mp \text { and } \bar{\sum}
$$

uniquely, since the operations of a C.A. are not uniquely determined by the partial ordering which they induce. However, there appears to be one natural definition, namely

$$
\sum_{i<\infty}\left\langle a_{i}, b_{i}\right\rangle=\langle a, b\rangle \text { where } a=\sum_{i<\infty} a_{i} \text { and } b=\sum_{a_{i}=a} b_{i} .
$$

As will be seen later this construction does not yield a C.A. unless some restrictive conditions are placed either on $\mathfrak{A}$ or else on some of the "summands" $\mathfrak{B}_{a}$.

In such directed sums, the element $\left\langle 0,0_{0}\right\rangle$ will act as zero element for $\mathfrak{E}$, where 0 is the zero element of $\mathfrak{A}$ and $0_{0}$ is the zero element of $\mathfrak{B}_{0}$. Consequently it seems natural to consider directed sums in which some of the summands are not C.A.'s but systems obtained from C.A.'s by removing the zero element. In some of the summands the presence of a zero element is clearly indicated. If $a$ is an element of $A$ such that there exist elements $a_{i} \in A$ for which

$$
a=\sum_{i<\infty} a_{i} \text { and } a_{i}<a \text { for each } i<\infty,
$$

and if $b_{i} \in B_{a_{i}}$ for $i<\infty$; then it would follow that

$$
\sum_{i<\infty}\left\langle a_{i}, b_{i}\right\rangle=\left\langle a, 0_{a}\right\rangle,
$$

where $0_{a}$ is the zero element of $\mathfrak{B}_{a}$. This suggests the following

Definition 3.1. Let

$$
\mathfrak{A}=\left\langle A,+, \sum\right\rangle
$$

be a C.A. An element $a \in A$ is said to be ACCESSIBLE in $\mathfrak{A}$ if and only if, there exist $a_{i} \in A, i<\infty$, for which

$$
a=\sum_{i<\infty} a_{i} \text { and } a_{i}<\text { a for every } i<\infty .
$$


Thus to say that an element is inaccessible means that it is indecomposable in terms of strictly smaller elements.

Definition 3.2. Let

$$
\mathfrak{U}=\left\langle A,+, \sum\right\rangle
$$

be a C.A. with zero element 0. By the POSITIVE PART of $\mathfrak{A}$ is meant the algebra $\left\langle A-\{0\},+, \sum\right\rangle$.

When speaking of the positive part of a C.A., it shall be understood that the C.A. has at least two members, so that the positive part is not empty. Notice that every C.A. can be considered as the positive part of another C.A.

Definition 3.3. Let

$$
\mathfrak{A}=\left\langle A,+, \sum\right\rangle
$$

be a C.A. with zero element 0 . For each $a \in A$ let

$$
\mathfrak{B}_{a}=\left\langle B_{a},+_{a}, \sum_{a}\right\rangle .
$$

If $a=0$ or if $a$ is accessible in $\mathfrak{A}$ assume that $\mathfrak{B}_{a}$ is a C.A. with zero element $0_{a}$, otherwise assume that $\mathfrak{B}_{a}$ is the positive part of a C.A. By the DIRECTED SUM of the algebras $\mathfrak{B}_{a}$ over $\mathfrak{A}$-in symbols

$$
\sum_{(a, \mathfrak{l})} \mathfrak{B}_{a}
$$

—is meant the algebra

$$
\mathfrak{S}=\langle C, \mp, \bar{\Sigma}\rangle
$$

where $C$ is the set of all ordered pairs $\langle a, b\rangle$ with $a \in A$ and $b \in B_{a}$, and where

$$
\sum_{i<\infty}:\left\langle a_{i}, b_{i}\right\rangle
$$

is defined to be the element $\langle a, b\rangle \in C$ with

$$
a=\sum_{i<\infty} a_{i} \text { and } b=\sum_{a_{i}=a} b_{i} .
$$

Finite addition $\mp$ is defined analogously.

We now inquire under what conditions this directed sum is a C.A. Before stating the answer to this question it is convenient to introduce the following

Definition 3.4. Let

$$
\mathfrak{A}=\left\langle A,+, \sum\right\rangle
$$

be a C.A. An element $a \in A$ is said to be SEPARATING in $\mathfrak{A}$ if and only if, for each $b \in A$, either $a \leqq b$ or $b \leqq a$.

Obviously a C.A. is linearly ordered if and only if each of its elements is separating.

THEOREM 3.5. Under the assumptions of (3.3), the directed sum $\mathfrak{E}$ of the 
algebras $\mathfrak{B}_{a}$ over $\mathfrak{A}$ is itself a C.A. if and only if, for each $a \in A, a$ is separating and idemmultiple in $\mathfrak{A}$, or $B_{a}$ consists of just one element.

Proof. First assume that $\mathbb{C}$ is a C.A. Consider an element $a \in A$ which is not idemmultiple, and suppose $\alpha, \alpha^{\prime} \in B_{a}$. Then $2 a=a+a$ is accessible in $\mathfrak{A}$, whence $0_{2 a}$ exists in $B_{2 a}$ and

$$
2\langle a, \alpha\rangle=\left\langle 2 a, 0_{2 a}\right\rangle=2\left\langle a, \alpha^{\prime}\right\rangle .
$$

By (2.6) this implies that $\langle a, \alpha\rangle=\left\langle a, \alpha^{\prime}\right\rangle$ and consequently $\alpha=\alpha^{\prime}$. Thus $B_{a}$ consists of just one element.

Now suppose $a \in A$ is idemmultiple but not separating in $\mathfrak{A}$. There then exists an element $b \in A$ such that neither $a \leqq b$ nor $b \leqq a$. Then $a<a+b$ and $b<a+b$ so that $a+b$ is accessible in $\mathfrak{A}$. Consequently $0_{a+b}$ exists in $B_{a+b}$. Choose a fixed element $\beta \in B_{b}$. For any elements $\alpha, \alpha^{\prime} \in B_{a}$ one then has

$$
\langle a, \alpha\rangle \mp\langle b, \beta\rangle=\left\langle a+b, 0_{a+b}\right\rangle=\left\langle a, \alpha+{ }_{a} \alpha^{\prime}\right\rangle \mp\langle b, \beta\rangle \text {. }
$$

By (2.4) this implies the existence of elements $\langle c, \gamma\rangle,\left\langle a_{1}, \alpha_{1}\right\rangle,\left\langle a_{2}, \alpha_{2}\right\rangle \in C$ such that

$$
\begin{aligned}
\langle a, \alpha\rangle & =\langle c, \gamma\rangle \mp\left\langle a_{1}, \alpha_{1}\right\rangle, \\
\left\langle a, \alpha+{ }_{a} \alpha^{\prime}\right\rangle & =\langle c, \gamma\rangle \mp\left\langle a_{2}, \alpha_{2}\right\rangle, \\
\langle b, \beta\rangle \mp\left\langle a_{1}, \alpha_{1}\right\rangle & =\langle b, \beta\rangle=\langle b, \beta\rangle \mp\left\langle a_{2}, \alpha_{2}\right\rangle .
\end{aligned}
$$

These equations imply that $a=c+a_{1}, a=c+a_{2}$, and $b+a_{1}=b=b+a_{2}$. Consequently $a_{1} \leqq a, a_{2} \leqq a, a_{1} \leqq b$, and $a_{2} \leqq b$. But $a_{1} \neq a \neq a_{2}$ by the definition of $b$, whence $a_{1}<a$ and $a_{2}<a$. It follows that either $c=a$ and $\alpha=\gamma=\alpha+{ }_{a} \alpha^{\prime}$, or else $c<a$ and $\alpha=0_{a}=\alpha+{ }_{a} \alpha^{\prime}$. In either case one has $\alpha^{\prime} \leqq{ }_{a} \alpha$. Similarly $\alpha \leqq \alpha^{\prime}$, whence $\alpha=\alpha^{\prime}$. Thus $B_{a}$ consists of just one element. This concludes the proof of the forward implication.

In order to prove the reverse implication it is necessary to check that Axioms I-VII for a C.A. are satisfied. A number of special cases must be considered and the checking, while straightforward, is tedious. Consequently this part of the proof is omitted.

The problem of representing an arbitrary C.A. as a directed sum will be considered next. Since (3.5) gives assurance that in any such representation the only nontrivial summand algebras will be those corresponding to idemmultiple separating elements of the index algebra, it seems natural to consider directed sums in which every element of the index algebra is idemmultiple and separating. Hence, as essentially the most important case, one should study directed sums over a linearly ordered idemmultiple C.A.

Definition 3.6. If $\mathfrak{A}$ is a C.A., $\mathfrak{B}$ is the positive part of $a$ C.A., then

$$
\mathfrak{A} \oplus \mathfrak{B}=\sum_{(c, \mathbb{E})} \mathfrak{E}_{c},
$$


where $\mathbb{E}$ is the C.A. of order 2 containing only the elements 0 and $\infty, \mathbb{S}_{0}=\mathfrak{A}$, $\mathfrak{S}_{\infty}=\mathfrak{B}$.

Thus every element of $\mathfrak{A}$ is less than every element of $\mathfrak{B}$ in this sum.

Definition 3.7. Let $\mathfrak{A}$ be a $C$.A. Then $\mathfrak{A}$ is said to be LINEARLy INDEComPOSABLE if and only if, whenever, $\mathfrak{U} \cong \mathfrak{B} \oplus \mathfrak{S}$, then $\mathfrak{B}$ must be a trivial one-element algebra.

Definition 3.8. Let

$$
\mathfrak{A}=\left\langle A,+, \sum\right\rangle
$$

be a C.A., $B$ an ideal in $\mathfrak{A}$. Then $B$ is said to be a SEPARATING IDEAL of $\mathfrak{A}$ if and only if, $b \in B$ and $a \in A-B$ implies that $b<a$.

Theorem 3.9. Let $\mathfrak{A}$ be a C.A., $B$ an ideal in $\mathfrak{A}$. If $B$ is a separating ideal of $\mathfrak{A}$, then $B$ is a separating element in the C.A. $\Im(\mathfrak{A})$.

Proof. Trivial.

It is possible to show by a counter-example that the converse to (3.9) does not hold.

THEOREM 3.10. Let

$$
\mathfrak{A}=\left\langle A,+, \sum\right\rangle
$$

be a C.A., $B$ a separating ideal in $\mathfrak{A}$. Then:

(i) For every $x, y \in A, x \equiv_{B} y$ if, and only if, either $x, y \in B$ or $x=y$.

(ii) The algebra

$$
\mathfrak{A}^{\prime}=\langle(A-B) \cup\{0\},+, \Sigma\rangle
$$

is a cardinal subalgebra of $\mathfrak{A}$, and

$$
\mathfrak{A}^{\prime} \cong \mathfrak{A} / B
$$

(iii) $\mathfrak{A} \cong \mathfrak{B} \oplus \mathbb{R}$, where $\mathfrak{B}=\langle B,+, \Sigma\rangle, \mathfrak{E}=\langle A-B,+, \Sigma\rangle$.

Proof. (i) If $x, y \in B$ or $x=y$, then obviously $x \equiv_{B} y$. If $x \in A-B, b \in B$, then $x>\infty b \in B$, since $B$ is a separating ideal and hence $x+b=x$. It follows that if $x \in A-B, x \equiv_{B} y$, then $x=y$.

(ii) Follows immediately from (i).

(iii) From (i) and (ii) one sees that the mapping $\langle u, a\rangle \rightarrow a$ gives an isomorphism of $\mathfrak{B} \oplus \mathfrak{C}$ onto $\mathfrak{A}$.

The concept of a separating ideal gives an intrinsic characterization of a linearly indecomposable C.A. as follows:

THEOREM 3.11. Let

$$
\mathfrak{U}=\langle A,+, \Sigma\rangle
$$

be a C.A. Then $\mathfrak{A}$ is linearly indecomposable if, and only if, $\mathfrak{A}$ contains no separating ideals other than $A$ and $\{0\}$. 
Proof. If $\mathfrak{A}$ is linearly indecomposable, then it can contain no nontrivial separating ideal by (3.10).

Assume

$\mathfrak{B} \oplus \mathfrak{C} \cong \mathfrak{A}$

under an isomorphism $f$, where $\mathfrak{B}=\left\langle B,+^{\prime}, \sum^{\prime}\right\rangle$ is a C.A. and $\mathfrak{E}=\left\langle C,+^{\prime \prime}, \sum^{\prime \prime}\right\rangle$ is the positive part of a C.A. Let $B^{\prime}$ be the image of $\{\langle 0, b\rangle \mid b \in B\}$ under $f$. Obviously $B^{\prime}$ is an ideal in $\mathfrak{A}$. If $x \in A-B^{\prime}, y \in B^{\prime}$, then there exist elements $b \in B, c \in C$ such that $x=f(\langle 0, b\rangle), y=f(\langle\infty, c\rangle)$, whence $x\langle y$, since $\langle 0, b\rangle$ $\left\langle\langle\infty, c\rangle\right.$. Hence $B^{\prime}$ is a separating ideal of $\mathfrak{A}$. If $\mathfrak{A}$ contains no nontrivial separating ideals, $B^{\prime}=\{0\}$, and consequently $\mathfrak{A}$ is linearly indecomposable.

THEOREM 3.12. Let

$$
\mathfrak{U}=\left\langle A,+, \sum\right\rangle
$$

be a C.A., and let $K$ be the class of all separating ideals of $\mathfrak{A}$. If $\varnothing \neq L \subset K$, then

$$
\bigcap_{x \in L} X \in K \text { and } I\left(\bigcup_{X \in L} X\right) \in K .
$$

Proof. Obviously $\bigcap_{X \in L} X$ is an ideal. Assume that $x \in A-\bigcap_{X \in L} X$, $y \in \bigcap_{X \in L} X$. Then $x \in A-X$ and $y \in X$ for some $X \in L$. Hence $y<x$ and $\bigcap_{x \in L} X \in K$.

Assume $x \in A-I\left(\cup_{X \in L} X\right), y \in I\left(\cup_{X \in L} X\right)$. In this case $x \in A-X$ for each $X \in L$, and

$$
y=\sum_{i<\infty} y_{i}
$$

where, for each $i<\infty, y_{i} \in X_{i} \in L$. Since $K$ is a linearly ordered subset of $\Im(\mathfrak{A})$, one has, for each $n<\infty$,

$$
\sum_{i<n} y_{i} \in X_{j} \text { for some } j<n,
$$

whence $x>\sum_{i<n} y_{i}$ for each $n<\infty$. By (2.5), $x \geqq y$. Since $x \neq y$, it follows that $x>y$, and hence $I\left(\cup_{x \in L} X\right) \in K$.

Definition 3.13. Let

$$
\mathfrak{A}=\left\langle A,+, \sum\right\rangle
$$

be $a$ C.A., $a \in A$. Let $K$ be the class of all separating ideals $X$ of $\mathfrak{A}$ such that $a \in X$. Let $L$ be the class of all separating ideals $X$ of $\mathfrak{A}$ such that $a \notin X$. The quantities $M_{\mathfrak{I}}(a)$ and $m_{\mathfrak{A}}(a)$ are then defined by

$$
\begin{aligned}
& M_{\mathfrak{I}}(a)=\bigcap_{\boldsymbol{X} \in K} X, \\
& m_{\mathfrak{Y}}(a)=\bigcup_{X \in L} X .
\end{aligned}
$$


By (3.12), $M \mathfrak{q}(a)$ and $I(m \mathfrak{q}(a))$ are separating ideals of $\mathfrak{A}$. Obviously $a \in M \mathbf{a}(a)$.

Where no confusion would result the subscript $\mathfrak{A}$ will be dropped and the quantities denoted by $M(a)$ and $m(a)$.

The main theorem on representation of an arbitrary C.A. by a directed sum may now be stated. This theorem asserts that every C.A. has a unique representation as a linear idemmultiple directed sum of linearly indecomposable C.A.'s and their positive parts.

TheOREM 3.14. Let

$$
\mathfrak{U}=\langle A,+, \Sigma\rangle
$$

be a C.A. Then

$$
\mathfrak{A} \cong \sum_{(b, \mathfrak{B})} \mathfrak{B}_{b}
$$

where (i) $\mathfrak{B}$ is a linearly ordered idemmultiple C.A.,

(ii) each $\mathfrak{B}_{b}$ is the positive part of a linearly indecomposable C.A., and

(iii) if $b=0$ or if $b$ is accessible in $\mathfrak{B}$, then $\mathfrak{B}_{b}$ is a trivial one-element C.A.

Furthermore, the algebras $\mathfrak{B}, \mathfrak{B}_{b}$ are uniquely determined, up to isomorphism, by this representation and conditions (i), (ii) and (iii).

Proof. Let $B$ be the class of all separating ideals $X$ of $\mathfrak{A}$ such that $X=M(a)$ for some $a \in A$. For each $X \in B$, let

$$
B_{X}=\{a \mid a \in A \text { and } M(a)=X\} .
$$

First it will be shown that the algebras

$$
\mathfrak{B}=\left\langle B,+, \sum\right\rangle
$$

(considered as a subalgebra of the ideal algebra $\Im(\mathfrak{A})$ ), and

$$
\mathfrak{B}_{X}=\left\langle B_{X},+, \sum\right\rangle
$$

for $X \in B$, satisfy the conditions of the theorem.

To verify (i) it is sufficient to show that $B$ is closed under addition. Suppose $X_{i} \in B$ for $i<\infty$, and let

$$
X=\sum_{i<\infty} X_{i}=I\left(\bigcup_{i<\infty} X_{i}\right)
$$

By (3.12), $X$ is a separating ideal in $\mathfrak{A}$. For each $i<\infty$, choose elements $a_{i}$ such that $X_{i}=M\left(a_{i}\right)$, and let

$$
a=\sum_{i<\infty} a_{i}
$$

Obviously $a \in X$. If $Y$ is a separating ideal of $\mathfrak{A}$ such that $a \in Y$, then $a_{i} \in Y$ 
for each $i<\infty, X_{i} \subset Y$ for each $i<\infty$, and hence $X \subset Y$. Consequently $X=M(a) \in B$.

It is most convenient to check (iii) next. If $X=\{0\}$, then $B_{X}=\{0\}$ and the conclusion follows. Assume that $X$ is accessible in $\mathfrak{B}$. Hence

$$
X=\sum_{i<\infty} X_{i} \text { where } X>X_{i} \in B \text { for each } i<\infty .
$$

Choose elements $x, y \in B_{X}$ arbitrarily. Then

$$
x=\sum_{i<\infty} x_{i}, \quad \text { with } \quad x_{i} \in X_{i} \text { for } i<\infty .
$$

Since $B$ is linearly ordered, $\sum_{i<n} X_{i}=X_{j}<X$ for some $j<n$, and for each $n<\infty$. Hence $y \notin \sum_{i<n} X_{i}$ for each $n<\infty$, and

$$
\sum_{i<n} x_{i}<y \text { for each } n<\infty \text {. }
$$

By (2.5), $x \leqq y$. Symmetrically $y \leqq x$, and consequently $x=y$. We observe that this single element of $B_{X}$ is idemmultiple, whence (iii) follows.

In order to check (ii) one may now assume that $X=M(a) \in B$ is not equal to $\{0\}$ and is inaccessible in $\mathfrak{B}$, and proceed to show that the algebra

$$
\mathfrak{B}_{X}^{\prime}=\left\langle B_{X} \cup\{0\},+, \sum\right\rangle
$$

is a linearly indecomposable C.A.

Let $K$ be the family of all separating ideals $Y \in B$ with $Y<X$, and let

$$
Z=\underset{C \in K}{\bigcup} C .
$$

If $y_{i} \in Z$ for $i<\infty$, then for some $Y_{i} \in K$ we have $y_{i} \in Y_{i}<X$, whence $\sum y_{i} \in \sum Y_{i} \leqq X$. Equality is impossible since $X$ is inaccessible, and it follows that $\sum Y_{i} \in K, \sum Y_{i} \in Z$. This proves that $Z$ is closed under addition and consequently is itself a separating ideal, $Z \leqq X$. If $Z=X=M(a)$, then we would have $a=\sum y_{i}, y_{i} \in Y_{i} \in K, Y_{i}<X$. This would imply that $X=\sum Y_{i}$, again contradicting the inaccessibility of $X$. Thus $Z$ is a separating ideal, $Z<X$, and furthermore $B_{X}=X-Z$. Since $Z$ is also a separating ideal in $X$, (3.10) assures one that $\mathfrak{B}_{X}^{\prime}$ is a C.A.

To show that $\mathfrak{B}_{X}^{\prime}$ is linearly indecomposable, assume that $Y$ is a separating ideal in $\mathfrak{B}_{X}^{\prime}$. Then $Y \cup Z$ is a separating ideal in $\mathfrak{A}$ with $Y \cup Z \leqq X$. If $Y \cup Z=X$, then $Y=B_{X} \cup\{0\}$. If $Y \cup Z<X$, choose $y \in Y$ arbitrarily and we have $M(y) \leqq Y \cup Z<X$, whence $y \notin B_{X}, y=0$. Thus $Y=B_{X} \cup\{0\}$ or $Y=\{0\}$, and (3.11) requires that $\mathfrak{B}_{X}^{\prime}$ be linearly indecomposable. This completes the verification of (ii).

Let

$$
\mathfrak{l}=\langle C, \overline{+}, \bar{\Sigma}\rangle=\sum_{(X, \mathfrak{B})} \mathfrak{B}_{X}
$$


(3.5) implies that $\mathfrak{R}$ is a C.A., and it is now a simple matter to construct the isomorphism between $\mathfrak{A}$ and $\mathfrak{C}$. For $a \in A$, let

$$
f(a)=\langle M(a), a\rangle \in C .
$$

Clearly $f$ is a one-to-one function mapping $A$ onto $C$. For elements $a_{i} \in A$, $i<\infty$, and

$$
a=\sum_{i<\infty} a_{i},
$$

it is necessary to show that $f(a)=\bar{\sum} f\left(a_{i}\right)$, i.e.,

$$
M(a)=\sum_{i<\infty} M\left(a_{i}\right) \text { and } a=\sum_{M\left(a_{i}\right)=M(a)} a_{i} .
$$

- Obviously $M(a) \geqq \sum M\left(a_{i}\right)$ since the inequality holds for each index. But $a=\sum a_{i} \in \sum M\left(a_{i}\right)$, which implies that $M(a) \leqq \sum M\left(a_{i}\right)$, whence $M(a)$ $=\sum M\left(a_{i}\right)$. If $M\left(a_{i}\right)<M(a)$ for each $i<\infty$, then $M(a)$ is accessible in $\mathfrak{B}$, and (iii) implies that $a$ must be the zero element of $\mathfrak{B}_{M(a)}$, being its only element. If $M\left(a_{i}\right)=M(a)$ for some indices $i<\infty$, whenever $M\left(a_{j}\right)<M(a)=M\left(a_{i}\right)$ one has $\infty a_{j}<a_{i}$ and consequently $a_{i}+a_{j}=a_{i}$. Hence

$$
a=\sum_{i<\infty} a_{i}=\sum_{M\left(a_{i}\right)=M(a)} a_{i}+\sum_{M\left(a_{j}\right)<M(a)} a_{j}=\sum_{M\left(a_{i}\right)=M(a)} a_{i} .
$$

In every case it follows that

$$
a=\sum_{M\left(a_{i}\right)=M(a)} a_{i}
$$

and thus

$$
f\left(\sum_{i<\infty} a_{i}\right)=\sum_{i<\infty} f\left(a_{i}\right) .
$$

This proves that $f$ gives the required isomorphism.

The uniqueness of this representation follows by straightforward checking that in any isomorphism of $\mathfrak{A}$ onto a directed sum satisfying (i), (ii) and (iii), the counter images of the various "summands" are the algebras $B_{X}$.

This completes the proof of the theorem.

Definition 3.15. Let

$$
\mathfrak{U}=\left\langle A,+, \sum\right\rangle
$$

be a C.A. The subalgebras $B_{X}$ of $\mathfrak{A}$, defined in the proof of (3.14), will be termed the LINEARLY INDECOMPOSABLE SUMMANDS of $\mathfrak{A}$.

Theorem 3.16. Let

$$
\mathfrak{A}=\left\langle A,+, \sum\right\rangle
$$

be a C.A., $a \in A$. If $B_{X}$ is the linearly indecomposable summand of $\mathfrak{A}$ containing $a$, then 


$$
B_{X}=M(a)-m(a) .
$$

Proof. By definition $B_{X} \subset M(a)$. For $b \in A, b \in B_{X}$ if, and only if, $M(b)$ $=M(a) . M(b)=M(a)$ implies that $b \notin m(a)$, and hence $B_{X} \subset M(a)-m(a)$. If $b \in M(a)-m(a)$, then $M(b) \leqq M(a)$ but $M(b) \nless M(a)$, whence $M(a)-m(a)$ $C B_{X}$, and $B_{X}=M(a)-m(a)$.

The study of the structure of arbitrary C.A.'s has now been reduced to the study of linearly indecomposable C.A.'s. In the next section this theory will be applied to characterize certain classes of C.A.'s whose linearly indecomposable summands may be described more or less completely in terms of simple and idemmultiple C.A.'s.

4. Primary cardinal algebras. One of the most fundamental arithmetical theorems for C.A.'s is (2.6) which states that, for integers $n$ with $0<n<\infty$,

$$
n a \leqq n b \text { implies } a \leqq b .
$$

This cancellation law for multiples can be split into two parts, namely:

$$
n a=n b \text { implies } a=b,
$$

and

$$
n a<n b \text { implies } a<b .
$$

In the simplest examples of C.A.'s, one finds that the condition " $n<\infty$ " is necessary in the first of these two parts. However, in a great many elementary C.A.'s-idemmultiple, simple, linearly ordered, etc.,- - one finds that the second part holds for $n=\infty$ as well as $n<\infty$. Hence, in seeking to describe the structure of more complicated C.A.'s in terms of these elementary ones, it seems natural to study the behavior of elements and algebras for which the second part holds with $n=\infty\left({ }^{1}\right)$.

Definition 4.1. Let

$$
\mathfrak{A}=\left\langle A,+, \sum\right\rangle
$$

be a C.A., $a \in A$.

(i) $a$ is said to be PRIMARY in $\mathfrak{A}$ if,

$$
\infty b<\infty \text { implies that } b<a, \text { for } b \in A \text {. }
$$

(ii) $\mathfrak{A}$ is said to be a PRIMARY C.A. if each of its elements is primary.

Note that, trivially, every idemmultiple element of a C.A. is primary.

In this section it is shown (4.5) that for a primary C.A. the linearly indecomposable summands are either idemmultiple or simple algebras. Consequently, for such algebras, Theorem 3.14 provides a fairly complete representation. Three lemmas are required.

(1) See Tarski [5] where algebras satisfying a similar but stronger property are discussed with special reference to the algebra of cardinal numbers. 
LEMMA 4.2. Let

$$
\mathfrak{A}=\left\langle A,+, \sum\right\rangle
$$

be a C.A., $a \in A$. Then $a$ is primary in $\mathfrak{A}$ if and only if, $\infty b<\infty a$ implies $\infty b<a$, for $b \in A$.

Proof. The reverse implication is obvious. Assume then that $a$ is primary and $b$ an element of $A$ such that $\infty b<\infty a$. Then $\infty(\infty b)=\infty b<\infty a$, whence $\infty b<a$.

LEMMA 4.3. Let

$$
\mathfrak{A}=\left\langle A,+, \sum\right\rangle
$$

be a C.A., and let $a, x$, and $y$ be nonzero elements of $A$, satisfying $a=x+y$.

If $a$ is primary and nonidemmultiple, then $x$ and $y$ must have a nonzero common lower bound.

Proof. If $a$ is not idemmultiple, the same must be true of either $x$ or $y$. Say $2 x>x$. Obviously $\infty x \leqq \infty a=\infty x+\infty y$. Assume first that $\infty x<\infty a$, whence by Lemma 4.2, $2 x \leqq \infty x<a=x+y$. Thus, for some $z \in A$, one would have $x+x+z=x+y$. By postulate VI for a C.A. one may write $x=x_{1}+y_{1}$ $=x_{2}+y_{2}, z=x_{3}+y_{3}$, where $x_{1}+x_{2}+x_{3}=x, y_{1}+y_{2}+y_{3}=y$. If $y_{1}=y_{2}=0$, then $x_{1}=x=x_{2}$, and $x=x_{1}+x_{2}+x_{3} \geqq 2 x$ contradicting the assumption that $x$ is not idemmultiple. Consequently $y_{1}$ and $y_{2}$ cannot both be zero, and one will be a nonzero lower bound for both $x$ and $y$. On the other hand, if $\infty x=\infty a$ $=\infty x+\infty y+y$, then, by postulate VI, one could write $y=\sum y_{i}$ where each $y_{i} \leqq x$. Some $y_{i}$ would again have to be nonzero, and thus in each case the existence of a nonzero lower bound for $x$ and $y$ has been demonstrated.

Lemma 4.4. Let

$$
\mathfrak{U}=\left\langle A,+, \sum\right\rangle
$$

be a primary C.A. If an element $a \in A$ is nonidemmultiple, then $\infty$ a is separating in $\mathfrak{A}$.

Proof. Assume $\infty a$ is not separating in $\mathfrak{A}$. This means that there exists an element $b \in A$ such that neither $b \leqq \infty a$ nor $\infty a \leqq b$, whence

$$
\infty a<\infty a+b \text {, and } b<\infty a+b .
$$

In this case $\infty a<\infty(a+b)$, which implies that $\infty a<a+b, a+b=\infty a+u$ $=a+\infty a+u=a+(a+b)$, for some element $u \in A$. Thus,

$$
2 a+b=a+b .
$$

By (2.3) there exist elements $c_{1}, c_{2}, c_{3}, c_{4} \in A$ such that 


$$
\begin{array}{rlrl}
2 a & =c_{1}+c_{2}, & a & =c_{1}+c_{3}, \\
b & =c_{3}+c_{4}, \quad b & =c_{2}+c_{4} .
\end{array}
$$

Obviously $\infty c_{2} \leqq \infty a$, but equality would imply that $\infty a \leqq \infty b$, which in turn would imply that $\infty a<b$ or $b \leqq \infty a$, contradicting the choice of $b$. Hence $\infty c_{2}<\infty a$, whence $\infty c_{2}<a$, and consequently

$$
a=\infty c_{2}+v=c_{2}+\infty c_{2}+v=c_{2}+a,
$$

for some element $v \in A$. Similarly $a=c_{3}+a$. Hence

$$
2 a=c_{1}+c_{3}+a=c_{1}+a=c_{1}+c_{2}+a=2 a+a=3 a .
$$

By (2.7), this implies that $a$ is idemmultiple, contradicting the hypothesis. It follows that no such element $b$ can exist, and the lemma is proved.

In view of Theorem 3.14, in order to determine the structure of primary C.A.'s it is necessary only to study linearly indecomposable primary C.A.'s. The next theorem describes such C.A.'s.

Theorem 4.5. Every linearly indecomposable primary C.A. is either simple or idemmultiple.

Proof. Let

$$
\mathfrak{A}=\left\langle A,+, \sum\right\rangle
$$

be a linearly indecomposable primary C.A., and assume that $\mathfrak{A}$ is not idemmultiple. Let $a$ be an arbitrary nonidemmultiple element of $\mathfrak{A}$. By (4.4) the ideal generated by $a, I(a)$, is a nonzero separating ideal in $\mathfrak{A}$, and by (3.11), $I(a)=A$.

Let

$$
B=\{x \mid x \in A \text { and } \infty x<\infty a\} .
$$

Obviously $y \leqq x \in B$ implies that $y \in B$, and to prove that $B$ is an ideal one need only show that it is closed under addition. Assume that $b_{i} \in B$ for $i<\infty$. Since, for $i<\infty, \infty b_{i}<a$, one has

$$
n \sum_{i<n} \infty b_{i}=\sum_{i<n} \infty b_{i} \leqq n a, \quad \text { for each } n<\infty .
$$

By (2.6), this implies that

$$
\sum_{i<n} \infty b_{i} \leqq a, \quad \text { for each } n<\infty,
$$

and by (2.5),

$$
\infty \sum_{i<\infty} b_{i}=\sum_{i<\infty} \infty b_{i} \leqq a .
$$

Equality is impossible, since $\infty \sum_{i<\infty} b_{i}$ is idemmultiple while $a$ is not. Hence $\sum_{i<\infty} b_{i} \in B$. This proves that $B$ is an ideal in $\mathfrak{A}$. If $x \in B$ then $\infty x=\infty a$, 
whence $b<x$ for each $b \in B$. Thus $B$ is a separating ideal in $\mathfrak{A}$, and, by (3.11), $B=\{0\}$. Consequently $\infty x=\infty a$ for each nonzero element $x \in A$. Thus $\mathfrak{A}$ can have no proper ideals and must be simple.

Combining (3.14) and (4.5), one obtains the following general representation theorem for primary C.A.'s.

Theorem 4.6. Let $\mathfrak{A}=\left\langle A,+, \sum\right\rangle$ be a primary C.A. Then

$$
\mathfrak{A} \cong \sum_{(b, \mathfrak{B})} \mathfrak{B}_{b}
$$

where (i) $\mathfrak{B}=\left\langle B,+, \sum\right\rangle$ is a linearly ordered idemmultiple $C . A$.;

(ii) for each $b \in B, \mathfrak{B}_{b}$ is the positive part of a simple C.A. or of a linearly indecomposable idemmultiple C.A.;

(iii) for each $b \in B$, if $b=0$ or if $b$ is accessible in $\mathfrak{B}$, then $\mathfrak{B}_{b}$ contains exactly one element.

The algebras $\mathfrak{B}$ and $\mathfrak{B}_{b}$, for $b \in B$, are uniquely determined (up to isomorphism) by $\mathfrak{A}$ and conditions (i)-(iii).

Theorem 4.6 reduces the study of primary C.A.'s to that of idemmultiple and simple algebras. As mentioned in $\$ 1$, every idemmultiple C.A. can be realized as an algebra of sets. However the structure theory of simple C.A.'s is not as satisfactory. The only known examples of simple C.A.'s are the algebra $\Re$ of non-negative real numbers (with $\infty$ added) and its three subalgebras: the non-negative integers (with $\infty$ added), the two-element algebra $\{0, \infty\}$, and the trivial algebra $\{0\}$. The problem of determining whether these are the only such algebras is still unsolved. In view of the preceding structure theorem, a definite answer to this question would be of importance. The following theorem fits rather naturally into the present development. It gives a fairly simple necessary and sufficient condition for a simple C.A. to be one of the above four, namely that its ordering be "Archimedian."

Theorem 4.7. Let

$$
\mathfrak{A}=\left\langle A,+, \sum\right\rangle
$$

be a simple C.A. having the property that, for each pair of elements $a, b \in A$, if $a$ is finite and $b \neq 0$, then $a \leqq n b$ for some integer $n<\infty$. Then $\mathfrak{A}$ is isomorphic to one of the four subalgebras of the algebra $\Re$ of non-negative real numbers (with $\infty$ added).

Proof. See reference [2].

In order to fit this theorem into the theory of this section one makes the following definition:

Definition 4.8. Let

$$
\mathfrak{A}=\left\langle A,+, \sum\right\rangle
$$

be a C.A. An element $a \in A$ is said to be STRONGLY PRIMARY if, for each $b \in A$, $b<\infty a$ implies that $b \leqq n a$ for some integer $n<\infty$. 
ThEOREM 4.9. Let

$$
\mathfrak{A}=\left\langle A,+, \sum\right\rangle
$$

be a C.A. Then every strongly primary element of $A$ is also primary.

Proof. Let $a$ be strongly primary, and let $b$ be an element of $A$ such that $\infty b<\infty a$. Then by the definition of strongly primary, $\infty b \leqq n a$ for some integer $n<\infty . \infty b=n(\infty b)$, and consequently (2.6) implies that $\infty b \leqq a$, whence $b \leqq a$. Thus $a$ is primary.

In view of theorems (4.7) and (4.9) one sees that if, in the statements of all theorems and definitions of this section one replaces the word "primary" by "strongly primary", and the phrase "simple C.A." by "cardinal subalgebra of $\Re$ ", all theorems will remain valid.

5. Star-products of cardinal algebras. One readily observes that the direct product of two nontrivial C.A.'s is always linearly indecomposable. Consequently the theory of the preceding two sections cannot be applied to such products. In analogy with the construction of semi-simple rings from simple rings one might ask whether there exist intrinsic characterizations for algebras which are built up as direct products of C.A.'s for which the directed sum decomposition gives an adequate description (e.g. of primary algebras). In a direct product of primary C.A.'s, for instance, the only nonidemmultiple primary elements are those of the factors, and such elements may be characterized as those nonidemmultiple elements of the product which cannot be written as sums of "disjoint" elements, (i.e., elements which have no nonzero common lower bound, see Definition 6.1). One can then ask whether the condition that all such elements be primary characterizes the class of all C.A.'s which are direct products of primary C.A.'s.

Consideration of such questions as these leads naturally to the study of a certain operation on C.A.'s which is at once a generalization of both a direct product and a directed sum. This operation turns out to be an extremely useful and suggestive tool, and is deserving of more detailed consideration. Theorem 5.1 and Definition 5.2 are the only parts of this section essential to the development of the theory of $\S 6$.

THEOREM 5.1. Let

$$
\mathfrak{A}=\left\langle A,+, \sum\right\rangle \text { and } \mathfrak{B}=\left\langle B,+^{\prime}, \sum^{\prime}\right\rangle
$$

be C.A.'s, and let $\phi$ be a homomorphism of $\mathfrak{B}$ into the ideal algebra $\Im(\mathfrak{A})$. Define the relation $R$ on $A \times B$ as follows: for $\langle a, b\rangle,\left\langle a^{\prime}, b^{\prime}\right\rangle \in A \times B$, define

$$
\langle a, b\rangle R\left\langle a^{\prime}, b^{\prime}\right\rangle
$$

if, and only if, $b=b^{\prime}$ and $a \equiv_{\phi(b)} a^{\prime}$, (see definition following (2.7)). Then $R$ is an infinitely additive equivalence relation over $\mathfrak{A} \times \mathfrak{B}$, and $(\mathfrak{A} \times \mathfrak{B}) / R$ is a C.A. 
Proof. $R$ is obviously an equivalence relation. In order to show that $R$ is infinitely additive, choose elements $\left\langle a_{i}, b_{i}\right\rangle,\left\langle a_{i}^{\prime}, b_{i}^{\prime}\right\rangle \in A \times B$ with $\left\langle a_{i}, b_{i}\right\rangle R$ $\left\langle a_{i}^{\prime}, b_{i}^{\prime}\right\rangle$, for $i<\infty$. Hence, for $i<\infty, b_{i}=b_{i}^{\prime}$ and there exist elements $x_{i}, y_{i} \in \phi\left(b_{i}\right)$ such that

$$
a_{i}+x_{i}=a_{i}^{\prime}+y_{i}
$$

Hence

$$
\begin{aligned}
\sum_{i<\infty}^{\prime} b_{i} & =\sum_{i<\infty}^{\prime} b_{i}^{\prime}, \\
\sum_{i<\infty} a_{i}+\sum_{i<\infty} x_{i} & =\sum_{i<\infty} a_{i}^{\prime}+\sum_{i<\infty} y_{i},
\end{aligned}
$$

and $\sum_{i<\infty} x_{i}, \sum_{i<\infty} y_{i} \in \sum_{i<\infty} \phi\left(b_{i}\right)=\phi\left(\sum_{i<\infty}^{\prime} b_{i}\right)$. Consequently

$$
\sum_{i<\infty}\left\langle a_{i}, b_{i}\right\rangle R \sum_{i<\infty}\left\langle a_{i}^{\prime}, b_{i}^{\prime}\right\rangle .
$$

This shows that $R$ is infinitely additive.

The algebra $(\mathfrak{A} \times \mathfrak{B}) / R$ obviously satisfies Postulates $I-V$ for a C.A., (2.1). Choose elements $\langle a, b\rangle,\left\langle a^{\prime}, b^{\prime}\right\rangle \in A \times B$ and $\left\langle a_{i}^{\prime \prime}, b_{i}^{\prime \prime}\right\rangle \in A \times B$ for $i<\infty$, such that

$$
\left(\langle a, b\rangle+\left\langle a^{\prime}, b^{\prime}\right\rangle\right) R \sum_{i<\infty}\left\langle a_{i}^{\prime \prime}, b_{i}^{\prime \prime}\right\rangle .
$$

This means that

$$
b+{ }^{\prime} b^{\prime}=\sum_{i<\infty}^{\prime} b_{i}^{\prime \prime},
$$

and that there exist elements $x, y \in \phi\left(b+^{\prime} b^{\prime}\right)$ such that

$$
a+a^{\prime}+x=\sum_{i<\infty} a_{i}^{\prime \prime}+y \text {. }
$$

Since

$$
\phi\left(b+{ }^{\prime} b^{\prime}\right)=\phi(b)+\phi\left(b^{\prime}\right)=\sum_{i<\infty} \phi\left(b_{i}^{\prime \prime}\right),
$$

one can find elements $z \in \phi(b), z^{\prime} \in \phi\left(b^{\prime}\right)$, and $y_{i} \in \phi\left(b_{i}^{\prime \prime}\right)$ for $i<\infty$, such that

$$
x=z+z^{\prime}, \quad y=\sum_{i<\infty} y_{i} \text {. }
$$

Hence

$$
(a+z)+\left(a^{\prime}+z^{\prime}\right)=\sum_{i<\infty}\left(a_{i}^{\prime \prime}+y_{i}\right) .
$$

By Postulate VI one can choose elements $a_{i}, a_{i}^{\prime} \in A$ for $i<\infty$, such that

$$
a+z=\sum_{i<\infty} a_{i}, \quad a^{\prime}+z^{\prime}=\sum_{i<\infty} a_{i}^{\prime},
$$


and

$$
a_{i}^{\prime \prime}+y_{i}=a_{i}+a_{i}^{\prime} \text { for } i<\infty .
$$

One can also choose elements $b_{i}, b_{i}^{\prime} \in B$ for $i<\infty$, such that

$$
b=\sum_{i<\infty}^{\prime} b_{i}, \quad b^{\prime}=\sum_{i<\infty}^{\prime} b_{i}^{\prime},
$$

and

$$
b_{i}^{\prime \prime}=b_{i}+^{\prime} b_{i}^{\prime} \text { for } i<\infty \text {. }
$$

Hence

$$
\langle a, b\rangle R \sum_{i<\infty}\left\langle a_{i}, b_{i}\right\rangle, \quad\left\langle a^{\prime}, b^{\prime}\right\rangle R \sum_{i<\infty}\left\langle a_{i}^{\prime}, b_{i}^{\prime}\right\rangle
$$

and

$$
\left\langle a_{i}^{\prime \prime}, b_{i}^{\prime \prime}\right\rangle R\left(\left\langle a_{i}+b_{i}\right\rangle+\left\langle a_{i}^{\prime}, b_{i}^{\prime}\right\rangle\right) \text { for } i<\infty .
$$

This shows that $(\mathfrak{A} \times \mathfrak{B}) / R$ satisfies Postulate VI for a C.A.

For $n<\infty$, assume that $\left\langle a_{n}, b_{n}\right\rangle,\left\langle a_{n}^{\prime}, b_{n}^{\prime}\right\rangle \in A \times B$ such that

$$
\left\langle a_{n}, b_{n}\right\rangle R\left(\left\langle a_{n+1}, b_{n+1}\right\rangle+\left\langle a_{n}^{\prime}, b_{n}^{\prime}\right\rangle\right) ;
$$

this means that

$$
b_{n}=b_{n+1}+^{\prime} b_{n}^{\prime},
$$

and that there exist elements $x_{n}, y_{n} \in \phi\left(b_{n}\right)$ such that

$$
a_{n}+x_{n}=a_{n+1}+a_{n}^{\prime}+y_{n} \text {. }
$$

Note that

$$
\phi\left(b_{0}\right) \supset \phi\left(b_{1}\right) \supset \phi\left(b_{2}\right) \supset \cdots \cdot
$$

For $n<\infty$, elements $z_{n} \in \phi\left(b_{n+1}\right), z_{n}^{\prime} \in \phi\left(b_{n}^{\prime}\right)$ may be chosen such that

$$
y_{n}=z_{n}+z_{n}^{\prime},
$$

since $\phi\left(b_{n}\right)=\phi\left(b_{n+1}\right)+\phi\left(b_{n}{ }^{\prime}\right)$. Furthermore, since $z_{0} \in \phi\left(b_{1}\right)=\phi\left(b_{2}\right)+\phi\left(b_{1}^{\prime}\right)$, elements $u_{0} \in \phi\left(b_{2}\right), v_{0} \in \phi\left(b_{1}^{\prime}\right)$ may be chosen such that

$$
z_{0}=u_{0}+v_{0} .
$$

$z_{1}+u_{0} \in \phi\left(b_{2}\right)=\phi\left(b_{3}\right)+\phi\left(b_{2}^{\prime}\right)$, and consequently there exist elements $u_{1} \in \phi\left(b_{3}\right)$, $v_{1} \in \phi\left(b_{2}^{\prime}\right)$ such that

$$
z_{1}+u_{0}=u_{1}+v_{1}
$$

Proceeding by induction, for each $n<\infty$, elements $u_{n} \in \phi\left(b_{n+2}\right), v_{n} \in \phi\left(b_{n+1}^{\prime}\right)$ may be chosen such that 


$$
z_{n}+u_{n-1}=u_{n}+v_{n},
$$

where one defines $u_{-1}=v_{-1}=0$. For $n<\infty$, let

$$
\begin{aligned}
& x_{n}^{\prime}=\sum_{i<\infty} x_{n+i}+u_{n-1}+v_{n-1} \in \phi\left(b_{n}\right), \\
& y_{n}^{\prime}=v_{n-1}+z_{n}^{\prime} \in \phi\left(b_{n}^{\prime}\right) .
\end{aligned}
$$

Adding $\sum_{i<\infty} x_{n+i+1}+u_{n-1}+v_{n-1}$ to each side of $\left(^{*}\right)$, and recalling that $y_{n}$ $=z_{n}+z_{n}^{\prime}$, one obtains, for $n<\infty$,

$$
\begin{aligned}
a_{n}+x_{n}^{\prime} & =\left(a_{n+1}+\sum_{i<\infty} x_{n+1+i}+u_{n-1}\right)+\left(a_{n}^{\prime}+v_{n-1}\right)+y_{n} \\
& =\left(a_{n+1}+\sum_{i<\infty} x_{n+1+i}+u_{n-1}+z_{n}\right)+\left(a_{n}^{\prime}+v_{n-1}+z_{n}^{\prime}\right) \\
& =\left(a_{n+1}+\sum_{i<\infty} x_{n+1+i}+u_{n}+v_{n}\right)+\left(a_{n}^{\prime}+v_{n-1}+z_{n}^{\prime}\right) \\
& =\left(a_{n+1}+x_{n+1}^{\prime}\right)+\left(a_{n}^{\prime}+y_{n}^{\prime}\right) .
\end{aligned}
$$

Hence, by Postulate VII, there exists an element $a \in A$ such that

$$
a_{n}+x_{n}^{\prime}=a+\sum_{i<\infty}\left(a_{n+i}^{\prime}+y_{n+i}^{\prime}\right), \quad \text { for each } n<\infty .
$$

Also there exists an element $b \in B$ such that

$$
b_{n}=b+{ }^{\prime} \sum_{i<\infty}^{\prime} b_{n+i}^{\prime}, \quad \text { for each } n<\infty .
$$

Since $x_{n}^{\prime} \in \phi\left(b_{n}\right)$ and $\sum_{i<\infty} y_{n+i}^{\prime} \in \phi\left(b_{n}^{\prime}\right) \subset \phi\left(b_{n}\right)$, for $n<\infty$, one has

$$
\langle a, b\rangle+\sum_{i<\infty}\left\langle a_{n+i}^{\prime}, b_{n+i}^{\prime}\right\rangle=\left\langle a+\sum_{i<\infty} a_{n+i}^{\prime}, b_{n}\right\rangle R\langle a, b\rangle .
$$

This shows that $(\mathfrak{A} \times \mathfrak{B}) / R$ satisfies Postulate VII for a C.A., and completes the proof that it is itself a C.A.

Definition 5.2. Let $\mathfrak{A}, \mathfrak{B}, \phi$, and $R$ be as in Theorem (5.1). The C.A. $(\mathfrak{A} \times \mathfrak{B}) / R$ is termed the STAR-PRODUCT of $\mathfrak{A}$ and $\mathfrak{B}$ modulo $\phi$ and is denoted by

$$
\mathfrak{U} *_{\phi} \mathfrak{B} \text {. }
$$

The relation $R$ is said to be generated by $\phi$.

REMARKs. (i) Let $\phi$ be the special homomorphism with $\phi(0)=\{0\}, \phi(b)$ $=A$ for $b \neq 0$. Then

$$
\mathfrak{A} *_{\phi} \mathfrak{B} \cong \mathfrak{A} \oplus \mathfrak{B}^{\prime},
$$

where $\mathfrak{B}^{\prime}$ is the positive part of $\mathfrak{B}$. 
(ii) If $\phi(b)=\{0\}$ for all $b \in B$, then

$$
\mathfrak{A} *_{\phi} \mathfrak{B} \cong \mathfrak{A} \otimes \mathfrak{B} \text {. }
$$

Hence the operation $*_{\phi}$ can be considered to be a generalization of both a directed sum and a direct product.

(iii) If $\phi$ is a homomorphism of $\mathfrak{B}$ into $\Im(\mathfrak{A})$, let $\phi$ be the natural homomorphism of $\mathfrak{B}$ into $\mathfrak{Y}(\mathfrak{A} / \phi(0))$ induced by $\phi$. Then it is easy to see that

$$
\mathfrak{A} *_{\phi} \mathfrak{B} \cong(\mathfrak{A} / \phi(0)) *_{\bar{\phi}} \mathfrak{B} \text {. }
$$

Hence one can always reduce the operation $*_{\phi}$ to the case where $\phi(0)=\{0\}$.

(iv) Since $\Im(\mathfrak{A})$ is an idemmultiple C.A., $\phi(b)=\phi(\infty b)$. Thus $\phi$ is determined by its values on the subalgebra of all idemmultiple elements of $\mathfrak{B}$. Hence $\phi$ can be considered as a homomorphism between idemmultiple C.A.'s.

The operation $*_{\phi}$ has been defined as an "outer" product. It is natural to ask whether there exists a corresponding "inner" product. That is, if a C.A. $\mathfrak{A}$ is represented in the form $\mathfrak{B} *_{\phi} \mathfrak{C}$, are there natural projections of $\mathfrak{B}$ and $\mathfrak{E}$ into $\mathfrak{A}$, and can the class of all subalgebras of $\mathfrak{A}$ which are obtained by such projections be characterized intrinsically? The answer is contained in the following two theorems.

The symbol $A(x)$ is used in the sense of Tarski, i.e.

$$
A(x)=\{y \mid x+y=x\} .
$$

Theorem 5.3. Let $\mathfrak{A}=\left\langle A,+, \sum\right\rangle, \mathfrak{B}=\left\langle B,+^{\prime}, \sum^{\prime}\right\rangle$, and $\mathfrak{S}=\left\langle C,+^{\prime \prime}, \sum^{\prime \prime}\right\rangle$ be C.A.'s, let $\phi$ be a homomorphism of $\mathfrak{C}$ into $\Im(\mathfrak{B})$ such that $\phi(0)=\{0\}$, and assume

$$
\mathfrak{B} *_{\phi} \mathfrak{C} \cong \mathfrak{A},
$$

under the isomorphism $f$. Define

$$
\begin{aligned}
& B^{\prime}=\{f(\langle b, 0\rangle / R) \mid b \in B\}, \\
& C^{\prime}=\{f(\langle 0, c\rangle / R) \mid c \in C\},
\end{aligned}
$$

$R$ being the relation on $B \times C$ generated by $\phi$. For $c^{\prime} \in C^{\prime}$, define

$$
\phi^{\prime}\left(c^{\prime}\right)=\{f(\langle b, 0\rangle / R) \mid b \in \phi(c)\},
$$

where $c \in C$ such that $c^{\prime}=f(\langle 0, c\rangle / R)$. Then

(i) $B^{\prime}$ is an ideal in $\mathfrak{A}$.

(ii) Every coset of $\mathfrak{A} \bmod B^{\prime}$ contains precisely one element $c^{\prime}$ of $C^{\prime}$, with $c^{\prime} \leqq a$ for each element $a$ of the coset.

(iii) For $c^{\prime} \in C^{\prime}$,

$$
\phi^{\prime}\left(c^{\prime}\right)=A\left(c^{\prime}\right) \cap B^{\prime}=I\left(c^{\prime}\right) \cap B^{\prime} .
$$

Proof. (i) is obvious from the definition of $B^{\prime}$.

Choose $a \in A$ arbitrarily. Hence $a=f(\langle b, c\rangle / R)$ for some $b \in B, c \in C$. Let 


$$
\begin{aligned}
& b^{\prime}=f(\langle b, 0\rangle / R) \in B^{\prime}, \\
& c^{\prime}=f(\langle 0, c\rangle / R) \in C^{\prime} .
\end{aligned}
$$

Then $a=b^{\prime}+c^{\prime}$, and consequently $a \equiv{ }_{B^{\prime}} c^{\prime}$.

Now given elements $a \in A, c^{\prime} \in C^{\prime}$ such that $a \equiv_{B^{\prime}} c^{\prime}$, there exist elements $b, u, v \in B$, and $c, c_{0} \in C$ such that $a=f(\langle b, c\rangle / R), c^{\prime}=f\left(\left\langle 0, c_{0}\right\rangle / R\right)$ and

$$
f(\langle b, c\rangle / R)+f(\langle u, 0\rangle / R)=f\left(\left\langle 0, c_{0}\right\rangle / R\right)+f(\langle v, 0\rangle / R) .
$$

Hence, $f$ being an isomorphism and $R$ being additive,

$$
\left\langle b+^{\prime} u, c\right\rangle R\left\langle v, c_{0}\right\rangle \text {. }
$$

Consequently $c=c_{0}, c^{\prime} \leqq a$. This shows that (ii) is satisfied.

It remains only to check (iii).

Note that, since $\langle 0, c\rangle R\left\langle 0, c_{0}\right\rangle$ if, and only if, $c=c_{0}, \phi^{\prime}$ is well defined. Choose an element $c^{\prime} \in C^{\prime}$ arbitrarily. Thus $c^{\prime}=f(\langle 0, c\rangle / R)$ for some $c \in C$. By definition

$$
\phi^{\prime}\left(c^{\prime}\right) \subset B^{\prime} .
$$

Choose $b^{\prime} \in \phi^{\prime}\left(c^{\prime}\right)$. Consequently $b^{\prime}=f(\langle 0, b\rangle / R)$ for some $b \in \phi(c)$. Since $b \equiv_{\phi(c)} 0$, one has $\langle b, c\rangle R\langle 0, c\rangle$ and $b^{\prime}+c^{\prime}=f(\langle b, c\rangle / R)=f(\langle 0, c\rangle / R)=c^{\prime}$. Hence $b^{\prime} \in A\left(c^{\prime}\right) \subset I\left(c^{\prime}\right)$, and $\phi^{\prime}\left(c^{\prime}\right) \subset A\left(c^{\prime}\right) \cap B^{\prime} \subset I\left(c^{\prime}\right) \cap B^{\prime}$. Choose $b^{\prime} \in I\left(c^{\prime}\right) \cap B^{\prime}$. Consequently $b^{\prime}=f(\langle b, 0\rangle / R)$ for some $b \in B$, and

$$
\begin{aligned}
f(\langle b, \infty c\rangle / R) & =f(\langle b, 0\rangle / R)+f(\langle 0, \infty c\rangle / R) \\
& =b^{\prime}+\infty c^{\prime} \\
& =\infty c^{\prime}, \text { since } b^{\prime} \in I\left(c^{\prime}\right), \\
& =f(\langle 0, \infty c\rangle / R) .
\end{aligned}
$$

Thus $\langle b, \infty c\rangle R\langle 0, \infty c\rangle, b \equiv_{\phi(\infty c)} 0, b \in \phi(\infty c)=\infty \phi(c)=\phi(c)$, and consequently $b^{\prime} \in \phi^{\prime}\left(c^{\prime}\right)$. Therefore $I\left(c^{\prime}\right) \cap B^{\prime} \subset \phi^{\prime}\left(c^{\prime}\right)$, whence

$$
\phi^{\prime}\left(c^{\prime}\right)=A\left(c^{\prime}\right) \cap B^{\prime}=I\left(c^{\prime}\right) \cap B^{\prime},
$$

completing the proof of the theorem.

The next theorem states that conditions (i)-(iii) of the preceding theorem are the characterization which one is seeking.

Theorem 5.4. Let

$$
\mathfrak{A}=\left\langle A,+, \sum\right\rangle
$$

be a C.A. Let $B^{\prime}, C^{\prime}, \phi^{\prime}$ satisfy conditions (i)-(iii) of (5.3). Let

$$
\mathfrak{B}=\left\langle B^{\prime},+, \Sigma\right\rangle, \quad \mathfrak{c}=\left\langle C^{\prime},+, \Sigma\right\rangle .
$$

Then

(i) (S is a cardinal subalgebra of $\mathfrak{A}$. 
(ii) $\phi^{\prime}$ is a homomorphism of $\left(5\right.$ into $\Im(\mathfrak{B})$ such that $\phi^{\prime}(0)=\{0\}$.

(iii) For $c \in C^{\prime}$ and $b, b^{\prime} \in B^{\prime}$,

$$
b \equiv_{\phi^{\prime}(c)} b^{\prime}
$$

if and only if, $b+c=b^{\prime}+c$.

(iv) $\mathfrak{B} *_{\phi^{\prime}} \mathfrak{C} \cong \mathfrak{A}$, under the mapping $f$ where, for $b \in B^{\prime}, c \in C^{\prime}$,

$$
f(\langle b, c\rangle / R)=b+c,
$$

$R$ being the relation on $B^{\prime} \times C^{\prime}$ generated by $\phi^{\prime}$.

Proof. Since every coset of $\mathfrak{A} \bmod B^{\prime}$ contains precisely one element of $\mathfrak{F}$, to prove (i) it is sufficient to show that $C^{\prime}$ is closed under $\sum$. If this is the case, the mapping $c \rightarrow c / B^{\prime}$ gives an isomorphism of $\mathfrak{c}$ onto the C.A. $\mathfrak{A} / B^{\prime}$. Choose elements $c_{i} \in C^{\prime}$ for $i<\infty$, and let $c=\sum_{i<\infty} c_{i}$. Choose an element $a \in A$ with $a \equiv B_{B^{\prime}} c$. Hence there exist elements $u, v \in B^{\prime}$ such that $a+u=c+v=\sum_{i<\infty} c_{i}$ $+v$. There exist elements $a^{\prime}, u^{\prime}, a_{i}, u_{i} \in A$ for $i<\infty$ such that

$$
\begin{aligned}
v & =a^{\prime}+u^{\prime}, & c_{i} & =a_{i}+u_{i} \quad \text { for } i<\infty, \\
a & =\sum_{i<\infty} a_{i}+a^{\prime}, & u & =\sum_{i<\infty} u_{i}+u^{\prime} .
\end{aligned}
$$

Since $u_{i} \in B^{\prime}$ for $i<\infty, a_{i} \leqq c_{i} \equiv{ }_{B^{\prime}} a_{i}$, and since each $c_{i}$ is minimal in $c_{i} / B^{\prime}$, one has $a_{i}=c_{i}$ for $i<\infty$. Hence

$$
a=\sum_{i<\infty} c_{i}+u^{\prime}=c+u^{\prime}
$$

whence $c \leqq a$. This shows that $c$ is minimal in $c / B^{\prime}$, and $c \in C^{\prime}$.

(ii) is obvious from condition (iii) of (5.3), assumed to hold.

Turn now to (iii). Choose elements $b, b^{\prime} \in B^{\prime}, c \in C^{\prime}$, and assume that $b \equiv_{\phi^{\prime}(c)} b^{\prime}$. Hence there exist elements $u, v \in A(c) \cap B^{\prime}$ such that $b+u=b^{\prime}+v$. Since $c+u=c=c+v$, this implies that $b+c=b+u+c=b^{\prime}+v+c=b^{\prime}+c$. Conversely, assume that $b+c=b^{\prime}+c$. By (2.4) there exist elements $d, e, e^{\prime} \in A$ such that $b=d+e, b^{\prime}=d+e^{\prime}, c+e=c=c+e^{\prime}$. Hence $e, e^{\prime} \in \phi^{\prime}(c)$ and $b+e^{\prime}$ $=d+e+e^{\prime}=b^{\prime}+e$. This shows that $b \equiv_{\phi^{\prime}(c)} b^{\prime}$ and completes the proof of (iii).

(iii) implies that the mapping $f$ defined in (iv) is a function on $\left(B^{\prime} \times C^{\prime}\right) / R$. Choose elements $b, b^{\prime} \in B^{\prime}, c, c^{\prime} \in C^{\prime}$ such that

$$
b+c=b^{\prime}+c^{\prime} \text {. }
$$

Hence $c \equiv_{B^{\prime}} c^{\prime}$ and consequently $c=c^{\prime}$, since each coset contains only one element of $C^{\prime}$. By (iii) one has $b \equiv_{\phi^{\prime}(c)} b^{\prime}$, whence $\langle b, c\rangle R\left\langle b^{\prime}, c^{\prime}\right\rangle$. The function $f$ is thus one-to-one. If an element $a \in A$ is chosen arbitrarily, then there exists an element $c \in C^{\prime}$ such that

$$
a \equiv_{B^{\prime}} c, \quad c \leqq a,
$$


i.e. there exists $b \in B^{\prime}$ such that

$$
a=b+c .
$$

Hence $f$ is a one-to-one function from $\mathfrak{B} *_{\phi^{\prime}} \mathbb{\complement}$ onto $\mathfrak{A} . f$ is obviously additive, and thus is an isomorphism. This completes the proof of the theorem.

Given two C.A.'s $\mathfrak{B}$ and $\mathbb{S}$ one may ask, in analogy with the theory of group extensions: What is the class of C.A. extensions $\mathfrak{A}$ of $\mathfrak{B}$ such that $B$ is an ideal in $\mathfrak{A}$ and

$$
\mathfrak{A} / B \cong \mathbb{E} ?
$$

Theorems (5.3) and (5.4) answer this question in part. Let $E$ be the class of all extensions $\mathfrak{A}$ of $\mathfrak{B}$ such that

(i) $B$ is an ideal in $\mathfrak{A}$;

(ii) $\mathfrak{A} / B \cong \mathbb{C}$;

(iii) every coset of $\mathfrak{A} \bmod B$ contains an element $c$ such that $c \leqq a$ for each element $a$ of the coset;

(iv) if $c$ is such a minimal element, then

$$
A(c) \cap B=I(c) \cap B .
$$

Then $E$ is in one-to-one correspondence with the class of all homomorphisms $\phi$ of $(\mathbb{S}$ into $\Im(\mathfrak{B})$ such that $\phi(0)=\{0\}$.

Notice that property (iv) above is in a certain sense a generalization of the property of being primary. If $c$ is primary in $\mathfrak{A}$ and $c \neq 0, b \in I(c) \cap B$, then $\infty b<\infty c$, whence $\infty b<c, b+c=c, b \in A(c) \cap B$. Hence in this case property (iv) follows.

6. Semi-primary cardinal algebras. In a direct product of primary C.A.'s the only nonidemmultiple primary elements are those of the factors, and these may be characterized as those nonidemmultiple elements which are disjunctively indecomposable (see Definition 6.1 below) in the product algebra. One might suspect then that this property would characterize such algebras in some sense. On further investigation however one discovers that this property is more intimately connected with the general star-product of C.A.'s discussed in $\$ 5$ rather than with the ordinary direct product.

Definition 6.1. Let $\mathfrak{A}=\left\langle A,+, \sum\right\rangle$, be a C.A.

(i) Two elements $x, y \in A$ are said to be DISJoINT in $\mathfrak{A}$, in symbols, $x \wedge y=0$, if $z \leqq x$ and $z \leqq y$ implies that $z=0$.

(ii) An element $a \in A$ is said to be DISJUNCTIVELy INDECOMPOSABLE in $\mathfrak{A}$ if $a=x+y$ with $x \wedge y=0$ implies that $x=0$ or $y=0$.

Definition 6.2. $A$ C.A. $\mathfrak{A}=\left\langle A,+, \sum\right\rangle$ is said to be semi-PRIMARy if every disjunctively indecomposable element of $\mathfrak{A}$ is primary.

The fundamental lemma required for the study of such semi-primary C.A.'s is the following: 
Theorem 6.3. Let $\mathfrak{A}=\left\langle A,+, \sum\right\rangle$ be a semi-primary C.A., and let $a$ and $b$ be disjunctively indecomposable elements of $\mathfrak{A}$. Then one of the following cases must hold: either (i) $a \wedge b=0$,

or (ii) $\infty a \leqq \infty b$,

or (iii) $\infty b \leqq \infty a$,

or (iv) $a$ and $b$ are idemmultiple.

Proof. Assume that neither (i), (ii), nor (iii) holds. The theorem will be proved if one can show that (iv) must then hold. If $a+b=x+y$ where $x \wedge y$ $=0, x \neq 0 \neq y$, then by (2.3) there exist elements $c_{1}, c_{2}, c_{3}, c_{4} \in A$ such that

$$
a=c_{1}+c_{2}, \quad b=c_{3}+c_{4}, \quad x=c_{1}+c_{3}, \quad y=c_{2}+c_{4} .
$$

From $x \wedge y=0$ one sees that $c_{1} \wedge c_{2}=0=c_{3} \wedge c_{4}$. Since $a$ and $b$ are disjunctively indecomposable, $c_{1}=0$ or $c_{2}=0$, and $c_{3}=0$ or $c_{4}=0$. Since $x \neq 0 \neq y$, one has $a=x, b=y$, or $a=y, b=x$, whence $a \wedge b=0$, contradicting our assumption that (i) does not hold. Hence $a+b$ is disjunctively indecomposable, and consequently primary.

Next one shows that $a+b$ is idemmultiple: Obviously

$$
\infty a \leqq \infty(a+b), \quad \infty b \leqq \infty(a+b),
$$

but equality in either case would imply (ii) or (iii), contradicting the initial assumption of the proof. Hence

$$
\infty a<a+b, \quad \infty b<a+b,
$$

whence

$$
\infty(a+b) \leqq 2(a+b) .
$$

This shows that $a+b$ is idemmultiple.

Since $a+b \leqq 2 a+b \leqq 2(a+b)=a+b$, one has that

$$
2 a+b=a+b .
$$

Now choose elements $d_{1}, d_{2}, d_{3}, d_{4} \in A$ such that $2 a=d_{1}+d_{2}, b=d_{3}+d_{4}$, $a=d_{1}+d_{3}, \quad b=d_{2}+d_{4}$. Hence $\infty d_{2} \leqq \infty a$, but equality would imply that $\infty a \leqq \infty b$, contradicting the assumption. Consequently $\infty d_{2}<a$, whence $a+d_{2}$ $=a$. Similarly $a+d_{3}=a$. Thus

$$
2 a=d_{1}+d_{3}+a=d_{1}+a=d_{1}+d_{2}+a=3 a .
$$

By (2.7), $a$ is idemmultiple. Similarly $b$ is idemmultiple.

THeOREM 6.4. Let $\mathfrak{A}=\left\langle A,+, \sum\right\rangle$ be a semi-primary C.A. Then either $\mathfrak{A}$ is linearly indecomposable, or

$$
\mathfrak{A} \cong \mathfrak{B} \oplus \mathfrak{S}
$$

where (i) $\mathfrak{B}$ is a linearly indecomposable semi-primary C.A. 
(ii) (5) is the positive part of a primary C.A.

The algebras $\mathfrak{B}$ and $\mathfrak{E}$ are uniquely determined up to isomorphism by $\mathfrak{A}$ and conditions (i), (ii).

Proof. If $\mathfrak{A}$ is primary, the theorem is obvious. Assume $\mathfrak{A}$ is not primary. Choose an element $a \in A$ such that $a$ is nonprimary, and let

$$
\begin{aligned}
B & =M(a), & C & =A-B, \\
\mathfrak{B} & =\langle B,+, \Sigma\rangle, & \mathbb{S} & =\langle C,+, \Sigma\rangle .
\end{aligned}
$$

By (3.12), $B$ is a separating ideal of $\mathfrak{A}$, and by (3.10),

$$
\mathfrak{A} \cong \mathfrak{B} \oplus \mathfrak{S} \text {. }
$$

Next we show that if $x$ is a nonprimary element of $\mathfrak{A}$, then $m(x)=\{0\}$. Assume $x$ is nonprimary. Thus $x=y+z$ for some elements $y, z \in A$ with $y \wedge z=0$ and $y \neq 0 \neq z$. If $X$ is a separating ideal of $\mathfrak{A}$ with $x \in X$, then $y \in X, z \notin X$ would imply that $y<z$, a contradiction; similàrly $y \notin X, z \in X$ would give a contradiction. Thus $y>u, z>u$ for each $u \in X$, and hence $X=\{0\}$, since $y \wedge z=0$. Consequently $m(x)=\{0\}$.

In particular, $m(a)=\{0\}$, which shows that $B$ is a linearly indecomposable semi-primary C.A. Furthermore, $c \in C$ implies that

$$
m(c) \supset B \neq\{0\} \text {. }
$$

Hence $c$ must be primary and $\mathbb{C}$ is the positive part of a primary C.A.

The uniqueness of the algebras $\mathfrak{B}$ and $\mathfrak{E}$ follows from (3.15), $\mathfrak{B}$ being linearly indecomposable.

Definition 6.5. Let $\mathfrak{A}=\left\langle A,+, \sum\right\rangle$ be a C.A.

By the WIDTH of $\mathfrak{A}$-in symbols, w( $\mathfrak{H})$-is meant the l.u.b. of the cardinal numbers of all sets $X \subset A-\{0\}$, such that $x \wedge y=0$ for all $x, y \in X$ with $x \neq y$.

Theorem 6.6. Let $\mathfrak{A}=\left\langle A,+, \sum\right\rangle$ be a C.A., $w(\mathfrak{H})<\infty, a \in A$. Then there exists a unique set $U \subset A-\{0\}$ such that

(i) The number of elements in $U$ is $\leqq w(\mathfrak{A})$.

(ii) $a=\sum_{x \in U} x$.

(iii) If $x$ and $y$ are elements of $U$ with $x \neq y$, then $x \wedge y=0$.

(iv) Every element of $U$ is disjunctively indecomposable.

Proof. Since $w(\mathfrak{A})<\infty$, choose $U$ to be a set of maximum cardinality satisfying (ii) and (iii). $U$ must then satisfy (i). If, for some element $x \in U$, $x=y+z$ with $y \wedge z=0, y \neq 0 \neq z$, then the set $(U-\{x\}) \cup\{y, z\}$ would contradict the maximality of $U$, hence $U$ satisfies (iv) also. Assume $U^{\prime}$ also satisfies (i)-(iv). Then

$$
a=\sum_{x \in U} x=\sum_{y \in U^{\prime}} y
$$


By (2.3) one can choose elements $x_{y} \in A$, for $x \in U, y \in U^{\prime}$, such that

$$
y=\sum_{x \in U} x_{y} \text { for } y \in U^{\prime}, \quad x=\sum_{y \in U^{\prime}} x_{y} \text { for } x \in U .
$$

For $x, x^{\prime} \in U, y, y^{\prime} \in U^{\prime}, x \neq x^{\prime}, y \neq y^{\prime}$, one has $x \wedge x^{\prime}=0=y \wedge y^{\prime}$, and consequently $x_{y} \wedge x_{y}^{\prime}=0=x_{y} \wedge x_{y^{\prime}}$. By (iv) one sees that, for each $x \in U, x=y$ for some $y \in U^{\prime}$, and conversely. Thus $U=U^{\prime}$.

DeFinition 6.7. Under the hypotheses of (6.6), the set $U$ shall be denoted by $\lambda(a)$, and the elements of $\lambda(a)$ shall be called the DISJUNCTIVELY INDECOMPOSABLE SUMMANDS of $a$.

THEOREM 6.8. Let

$$
\mathfrak{U}=\left\langle A,+^{\prime}, \sum^{\prime}\right\rangle, \quad \mathfrak{B}=\left\langle B,+^{\prime \prime}, \sum^{\prime \prime}\right\rangle
$$

be C.A.'s, $\phi$ a homomorphism of $\mathfrak{B}$ into $\Im(\mathfrak{H})$ with $\phi(0)=\{0\}$. Then

$$
w\left(\mathfrak{H} *_{\phi} \mathfrak{B}\right)=w(\mathfrak{A})+w(\text { kernel } \phi) .
$$

Proof. Let

$$
\mathfrak{C}=\mathfrak{A} *_{\phi} \mathfrak{B}=\left\langle C,+, \sum\right\rangle .
$$

By Theorem (5.3) one may assume that $A$ and $B$ are subalgebras of $\mathfrak{\complement}, A$ an ideal, $b$ minimal in $b / A$ for each $b \in B$, and $\phi(b)=I(b) \cap A$ for each $b \in B$.

$$
A \cap \text { kernel } \phi=\{0\} \text {. }
$$

Consequently,

$$
w(\mathfrak{S}) \geqq w(\mathfrak{A})+w(\text { kernel } \phi) .
$$

Let $X$ be any set of mutually disjoint elements of $C$. For each element $x \in X$, by (5.3), $x=y+z, y \in A, z \in B$. One may assume that $y=0$ or $z=0$, since if $z \neq 0, x$ may be replaced by $z$. If $y=0$ and $\phi(z) \neq 0$, then $x$ may be replaced by any nonzero element of $\phi(z)=I(z) \cap B$. Hence one may assume that,

$$
X \subset A \cup \text { kernel } \phi \text {. }
$$

Consequently

$$
w(\mathfrak{S}) \leqq w(\mathfrak{H})+w(\text { kernel } \phi) .
$$

Corollary 6.9. Let

$$
\mathfrak{A}=\left\langle A,+^{\prime}, \sum^{\prime}\right\rangle, \quad \mathfrak{B}=\left\langle B,+^{\prime \prime}, \sum^{\prime \prime}\right\rangle
$$

be C.A.'s, $A \neq\{0\}, \mathfrak{B}^{\prime}$ the positive part of $\mathfrak{B}$. Then

(i) $w(\mathfrak{A} \times \mathfrak{B})=w(\mathfrak{A})+w(\mathfrak{B})$,

(ii) $w\left(\mathfrak{A} \oplus \mathfrak{B}^{\prime}\right)=w(\mathfrak{A})$.

Definition 6.10. Let $\mathfrak{A}=\left\langle A,+, \sum\right\rangle$ be a C.A. The set of all ideals $X$ of $\mathfrak{A}$ 
which are factors of $\mathfrak{A}$, i.e. such that $A=X \times Y$ for some ideal $Y$ of $\mathfrak{A}$, will be denoted by $\mathfrak{F}(\mathfrak{H})$.

Definition 6.11. Let

$$
\mathfrak{A}=\left\langle A,+, \sum\right\rangle
$$

be a C.A. $\mathfrak{A}$ is said to be STRICTLy Nonidemmultiple if, for each $X \in \mathfrak{F}(\mathfrak{A}), X$ $=I(N)$, where $N$ is the set of all nonidemmultiple elements of $X$.

THEOREM 6.12. Let $\mathfrak{A}=\left\langle A,+, \sum\right\rangle$ be a semi-primary C.A. with $w(\mathfrak{H})<\infty$. Then $\mathfrak{A} \cong \mathfrak{B}_{\phi} \mathfrak{C}$, where (i) $\mathfrak{B}$ is a strictly nonidemmultiple semi-primary C.A.,

(ii) 5 is an idemmultiple C.A.,

(iii) $\phi$ is a homomorphism of $\mathfrak{C}$ into $\mathfrak{F}(\mathfrak{B})$ such that $\phi(0)=\{0\}$.

This representation is unique up to isomorphism.

Proof. Let $P$ be the class of all disjunctively indecomposable, nonidemmultiple elements of $\mathfrak{A}$. Let

$$
\begin{aligned}
& B=I(P), \\
& C=\{a \mid a \in A \text { and } \lambda(a) \cap B=\varnothing\}, \\
& \mathfrak{B}=\left\langle B,+, \sum\right\rangle, \quad \mathbb{S}=\left\langle C,+, \sum\right\rangle .
\end{aligned}
$$

For each element $c \in C$, let $\phi(c)=I(c) \cap B$.

Assume that $X \in \mathfrak{F}(\mathfrak{B})$, and choose an element $x \in X$ arbitrarily. Since $B=I(P)$,

$$
x \leqq \sum_{i<n} \infty x_{i},
$$

where $x_{i} \in P$ for $i<n \leqq \infty$. Without loss in generality one can assume that $x \wedge x_{i} \neq 0$ for each $i<n$, and consequently $x_{i} \in X$ for $i<n$, since each $x_{i}$ is disjunctively indecomposable. Hence

$$
X=I(N),
$$

where $N$ is the set of all nonidemmultiple elements of $X$. Thus $\mathfrak{B}$ is a strictly nonidemmultiple, semi-primary C.A.

If $a$ is an element of $C$, then every disjunctively indecomposable summand of $a$ is idemmultiple. Thus (5 satisfies (ii).

Next one wishes to show that every coset of $\mathfrak{A} \bmod B$ contains precisely one element of $\mathfrak{E}$, which is minimal in it. Choose an element $a \in A$ arbitrarily, and let

$$
\begin{aligned}
U & =\lambda(a) \cap B, & V & =\lambda(a)-B, \\
b & =\sum_{x \in U} x, & c & =\sum_{y \in V} y .
\end{aligned}
$$

Hence $b \in B, c \in C$, and $a=b+c$, i.e.

$$
c \leqq a \equiv_{B} c .
$$


Assume that $c, c^{\prime} \in C$ with $c \equiv_{B} c^{\prime}$. There then exist elements $b, b^{\prime} \in B$ such that

$$
c+b=c^{\prime}+b^{\prime} \text {. }
$$

Since $y \in \lambda(b), y \wedge x \neq 0$ for some $x \in \lambda(c)$, implies that $\infty y<x, x+y=x$, by (6.3) and the definitions of $B$ and $C$, and consequently one has that

$$
c+b=\sum_{x \in \lambda(c)} x+\sum_{y \in \lambda(b)} y=\sum_{x \in \lambda(c)} x+\sum_{y \in \lambda(b) ; y \wedge c=0} y .
$$

Hence

$$
\lambda(c+b)=\lambda(c) \cup W,
$$

where $W=\lambda(b) \cap\{y \mid y \wedge c=0\}$. Similarly

$$
\lambda\left(c^{\prime}+b^{\prime}\right)=\lambda\left(c^{\prime}\right) \cup W^{\prime},
$$

where $W^{\prime}=\lambda\left(b^{\prime}\right) \cap\left\{y \mid y \wedge c^{\prime}=0\right\}$. Since

$$
\begin{aligned}
\lambda(b+c) & =\lambda\left(b^{\prime}+c^{\prime}\right), \\
\lambda(c), \lambda\left(c^{\prime}\right) & \subset C, \quad W, W^{\prime} \subset B, \quad B \cap C=\{0\},
\end{aligned}
$$

one has

$$
\lambda(c)=\lambda\left(c^{\prime}\right), \quad c=c^{\prime} .
$$

Consequently every coset of $\mathfrak{A} \bmod B$ contains precisely one element of $\mathfrak{E}$ and this element is minimal in the coset. $\mathbb{S}$ being idemmultiple, for each $c \in C$,

$$
\phi(c)=I(c) \cap B=A(c) \cap B .
$$

Hence, by Theorem (5.3)

$$
\mathfrak{A} \cong \mathfrak{B} *_{\phi} \mathfrak{S},
$$

and this representation is unique.

Choose an element $c \in C$, and let

$$
Y=\{y \mid y \in B \text { and } \lambda(y) \cap \phi(c)=\varnothing\} .
$$

$Y$ is thus an ideal in $\mathscr{B}$, and $\phi(c) \cap Y=\{0\}$. For each $b \in B$,

$$
b=\sum_{x \in \lambda(b)} x=\sum_{x \in \lambda(b) \cap \cap_{\phi(c)}} x+\sum_{x \in \lambda(b)-\phi(c)} x=b^{\prime}+b^{\prime \prime},
$$

where $b^{\prime} \in \phi(c), b^{\prime \prime} \in Y$. Thus $B=\phi(c) \times Y$, and consequently

$$
\phi(c) \in \mathfrak{F}(\mathfrak{B}) .
$$

This completes the proof of the theorem.

Theorem 6.13. Let

$$
\mathfrak{A}=\left\langle A,+, \sum\right\rangle
$$


be a C.A. with $w(\mathfrak{A})<\infty$. Then there exist unique ideals $A_{0}, A_{1}, \cdots, A_{m-1}$ such that

$$
A=\prod_{i<m} A_{i}
$$

where (i) $m \leqq w(\mathfrak{A})$

(ii) For $i<m, A_{i}$ is a directly indecomposable ideal in $\mathfrak{A}$.

Proof. By Tarski (10.16).

Theorems (6.4), (6.8), (6.12) and (6.13) can now be combined to give a general decomposition theorem for semi-primary C.A.'s of finite width.

Theorem 6.14. Let $\mathfrak{A}=\left\langle A,+, \sum\right\rangle$ be a semi-primary C.A. with $1<w(\mathfrak{A})$ $<\infty$. Then

$$
\begin{aligned}
\mathfrak{A} & \cong \mathfrak{A}_{0} \oplus \mathfrak{D}, \\
\mathfrak{A}_{0} & \cong \mathfrak{B} *_{\phi} \mathfrak{C},
\end{aligned}
$$

and

$$
\mathfrak{B}=\prod_{i<m} \mathfrak{B}_{i}
$$

where (i) $\mathfrak{A}_{0}$ is a linearly indecomposable, semi-primary C.A.

(ii) $\mathfrak{D}$ is the positive part of a primary C.A. which is trivial in case $\mathfrak{A}$ is linearly indecomposable.

(iii) (5) is an idemmultiple C.A.

(iv) $m \leqq w(\mathfrak{A})$.

(v) For $i<m, \mathfrak{B}_{i}$ is a strictly nonidemmultiple, directly indecomposable, semi-primary C.A.

(vi) $\phi$ is a homomorphism of $\mathbb{E}$ into $\mathfrak{F}(\mathfrak{B})$ with $\phi(0)=\{0\}$.

(vii) For $i<m$,

$$
w\left(\mathfrak{B}_{i}\right)<w(\mathfrak{A}) .
$$

Furthermore, conditions (i)-(vi) uniquely determine the algebras $\mathfrak{A}_{0}, \mathfrak{D}, \mathfrak{S}$, $\mathfrak{B}_{0}, \cdots, \mathfrak{B}_{m-1}$ (up to isomorphism), the homomorphism $\phi$, and the integer $m$.

Proof. All parts except (vii) follow from theorems (6.4), (6.8), (6.12) and (6.13).

If kernel $\phi \neq\{0\}$, or if $m>1$, (vii) follows, since by (6.8) and (6.9)

$$
w(\mathfrak{A})=\sum_{i<m} w\left(\mathfrak{B}_{i}\right)+w(\text { kernel } \phi) .
$$

Assume kernel $\phi=\{0\}, m=1$. Then, by (v) and (vi),

$$
\mathfrak{A}_{0} \cong \mathfrak{B} \oplus \mathfrak{S}^{\prime},
$$

where $\mathfrak{C}^{\prime}$ is the positive part of $\mathfrak{C}$. By (i), $\mathfrak{C}$ is trivial and $\mathfrak{A}_{0} \cong \mathfrak{B}$. 
Let $P$ be the set of all disjunctively indecomposable, nonidemmultiple elements of $\mathfrak{B}$. Choose an element $b \in B$ such that $w(I(b))$ is maximal. Let

$$
\begin{aligned}
& P^{\prime}=\{x \mid x \in P \text { and } x \wedge b \neq 0\}, \quad P^{\prime \prime}=P-P^{\prime}, \\
& X=I\left(P^{\prime}\right), \quad Y=I\left(P^{\prime \prime}\right) .
\end{aligned}
$$

Obviously,

$$
X+Y=I(P)=B .
$$

One now wishes to show that $X \cap Y=\{0\}$. It is sufficient to show, for each $x \in P^{\prime}, y \in P^{\prime \prime}$, that $x \wedge y=0$. Choose elements $x \in P^{\prime}, y \in P^{\prime \prime}$. Thus $x \wedge b \neq 0$, $y \wedge b=0$. Assume that $x \wedge y \neq 0$. By (6.3)

$$
\infty x \leqq \infty y \text { or } \infty y \leqq \infty x .
$$

But $\infty x \leqq \infty y$ would imply that $x \wedge b=0$, contradicting the hypothesis. Hence $\infty y \leqq \infty x$. By (6.3) again,

$$
\infty x \leqq \infty b \text { or } \infty b \leqq \infty x .
$$

However, $\infty x \leqq \infty b$ and $y \wedge b=0$ would imply that $x \wedge y=0$, again contradicting hypothesis; while $\infty b \leqq \infty x \geqq \infty y$ and $y \wedge b=0$ would give $w(I(x))$ $>w(I(b))$, contradicting the choice of $b$. Since in every case one arrives at a contradiction, one concludes that

$$
X \cap Y=\{0\}, \quad B=X \times Y .
$$

By (v), this gives $B=X, P=P^{\prime}$.

Choose an element $a \in B$ arbitrarily. If $x \in \lambda(a)$, then $x \wedge b \neq 0$, and hence, by $(6.3)$

$$
\infty x<\infty b \text {, or } \infty b \leqq \infty x \text {. }
$$

$\infty x<\infty b$ for each $x \in \lambda(a)$ would imply that $\infty a<\infty b$, while $\infty b \leqq \infty x$ for some $x \in \lambda(a)$ would imply that $\infty b \leqq \infty a$. Consequently

$$
Z=\{a \mid a \in B \text { and } \infty a<\infty b\}
$$

is a separating ideal in $\mathfrak{B}$. Since $w(\mathfrak{B})>1, Z \neq\{0\}$, and $\mathfrak{B}$ is linearly decomposable. However this contradicts (i), since $\mathfrak{B} \cong \mathfrak{H}_{0}$. Therefore, for $i<m$,

$$
w\left(\mathfrak{B}_{i}\right)<w(\mathfrak{H}),
$$

which completes the existence part of the theorem.

The uniqueness of $\mathfrak{A}_{0}$ and $\mathfrak{D}$ follows from (6.4), that of $\mathfrak{B}$, $\mathfrak{S}$ and $\phi$ follows from (6.12), while (6.13), together with the fact that any direct factor of a strictly nonidemmultiple semi-primary C.A. is of the same type, assures the uniqueness of $\mathfrak{B}_{0}, \cdots, \mathfrak{B}_{m-1}$ and $m$. Thus the theorem is proved.

This theorem gives a decomposition of a semi-primary C.A. of finite width in terms of such algebras of smaller width. By repeating the process 
one would finally arrive at a decomposition of a semi-primary C.A. of finite width in terms of semi-primary C.A.'s of width 1 . To complete the discussion therefore, we need only the following

THEOREM 6.15. Every semi-primary C.A. of width 1 is primary.

Proof. Since no two nonzero elements of such a C.A. are disjoint, every element must be disjunctively indecomposable and hence primary.

The results of Theorems 6.14 and 6.15 may be stated in a less complete but possibly more suggestive form as follows:

THEOREM 6.16. Let $K$ be the class of all semi-primary C.A.'s of finite width. Then $K$ is the smallest class of C.A.'s with the following properties:

(i) If $\mathfrak{A}$ is a primary C.A. of finite width, then $\mathfrak{A} \in K$.

(ii) If $\mathfrak{A} \cong \mathfrak{B} \in K$, then $\mathfrak{A} \in K$.

(iii) If $\mathfrak{A}, \mathfrak{B} \in K$, then $\mathfrak{A} \times \mathfrak{B} \in K$.

(iv) If $\mathfrak{A} \in K, \mathfrak{B}$ is a primary C.A., and $\phi$ is a homomorphism of $\mathfrak{B}$ into $\mathfrak{F}(\mathfrak{A})$ such that $w($ kernel $\phi)<\infty$, then

$$
\mathfrak{U} *_{\phi} \mathfrak{B} \in K \text {. }
$$

Proof. Theorems 6.14 and 6.15 give assurance that every semi-primary C.A. of finite width may be obtained from primary C.A.'s using the operations (ii)-(iv). One needs only to check now that the property of being semiprimary of finite width is preserved under these operations.

(ii) and (iii) are obvious.

Assume that

$$
\mathfrak{A}=\left\langle A,+^{\prime}, \sum^{\prime}\right\rangle
$$

is a semi-primary C.A., $w(\mathfrak{A})<\infty$,

$$
\mathfrak{B}=\left\langle B,+{ }^{\prime \prime}, \sum^{\prime \prime}\right\rangle
$$

is a primary C.A., and $\phi$ is a homomorphism of $\mathfrak{B}$ into $\mathfrak{F}(\mathfrak{A})$ such that $w($ kernel $\phi)<\infty$. Since $\phi(0) \in \mathfrak{F}(\mathfrak{H}), \mathfrak{H} / \phi(0)$ is isomorphic to an element of $\mathfrak{F}(\mathfrak{A})$ and hence is semi-primary. Consequently, without loss in generality, one may assume that $\phi(0)=\{0\}$. Let

$$
\mathfrak{C}=\left\langle C,+, \sum\right\rangle=\mathfrak{A} *_{\phi} \mathfrak{B} .
$$

By (5.3), one may assume that $A$ is an ideal in $\mathfrak{c}, b \in B$ if, and only if, $b \in C$, $b$ minimal in $b / A$, and

$$
\phi(b)=I(b) \cap A=A(b) \cap A .
$$

Furthermore, since $\phi(b) \in \mathfrak{F}(\mathfrak{H})$ for $b \in B$, each element $c \in C$ may be represented as

$$
c=a+b
$$


where $a \in A, b \in B$, and $a$ is an element of the ideal complement of $\phi(b)$, i.e. $a \wedge b=0$. Hence every disjunctively indecomposable element of $\mathbb{5}$ is an element of $A$ or $B$.

If $a$ is a disjunctively indecomposable element of $A$, then $a$ is primary in $A$, whence $a$ is primary in $\mathfrak{E}, A$ being an ideal in $\mathfrak{E}$. Choose elements $b \in B$, $c \in C$ arbitrarily such that

$$
\infty c<\infty b \text {. }
$$

Choose elements $a^{\prime} \in A, b^{\prime} \in B$ such that $c=a^{\prime}+b^{\prime}$. Then

$$
\infty b^{\prime} \leqq \infty a^{\prime}+\infty b^{\prime}<\infty b .
$$

Since $\mathscr{B}$ is primary, one has $b^{\prime}<b$, while $\infty a^{\prime}<\infty b$ implies that

$$
a^{\prime} \in I(b) \cap A=\phi(b)=A(b) \cap A .
$$

Consequently,

$$
c=a^{\prime}+b^{\prime}<b .
$$

Thus every disjunctively indecomposable element of $\sqrt{5}$ is primary, i.e. $\sqrt{5}$ is semi-primary.

$$
w(\mathfrak{S})=w(\mathfrak{A})+w(\text { kernel } \phi)<\infty .
$$

This completes the proof of the theorem.

\section{REFERENCES}

1. G. Birkhoff, Generalized arithmetic, Duke Math. J. vol. 9 (1942) pp. 283-302.

2. A. B. Clarke, $A$ theorem on simple cardinal algebras, Michigan Math. J. vol. 3 (1956) pp. 113-116. $1-43$.

3. M. M. Day, Arithmetic of ordered systems, Trans. Amer. Math. Soc. vol. 58 (1945) pp.

4. A. Tarski, Cardinal algebras, New York, Oxford University Press, 1949.

5. - - Axiomatic and algebraic aspects of two theorems on sums of cardinals, Fund. Math. vol. 35 (1948) pp. 79-104.

University of Michigan, ANN ARbor, Mich. 